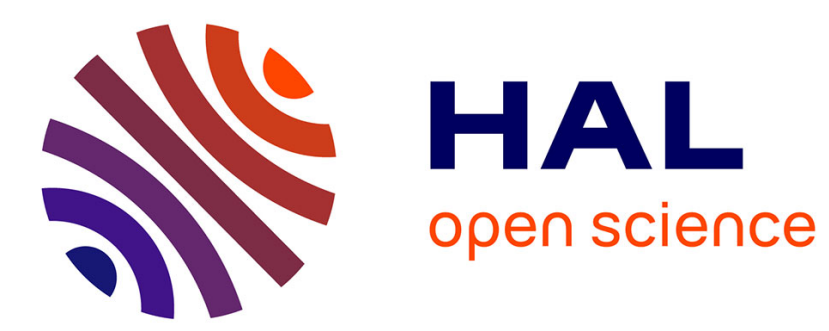

\title{
La légitime défense, hier et aujourd'hui : le " résidu réaliste " du droit international ?
}

Thierry Ménissier

\section{To cite this version:}

Thierry Ménissier. La légitime défense, hier et aujourd'hui : le " résidu réaliste " du droit international ?. Revue de Métaphysique et de Morale, 2009, 64 (4), pp.443-458. 10.3917/rmm.094.0951. halshs-01652821

\section{HAL Id: halshs-01652821 \\ https://shs.hal.science/halshs-01652821}

Submitted on 1 Dec 2017

HAL is a multi-disciplinary open access archive for the deposit and dissemination of scientific research documents, whether they are published or not. The documents may come from teaching and research institutions in France or abroad, or from public or private research centers.
L'archive ouverte pluridisciplinaire HAL, est destinée au dépôt et à la diffusion de documents scientifiques de niveau recherche, publiés ou non, émanant des établissements d'enseignement et de recherche français ou étrangers, des laboratoires publics ou privés. 


\title{
LA LÉGITIME DÉFENSE, HIER ET AUJOURD'HUI : LE « RÉSIDU RÉALISTE » DU DROIT INTERNATIONAL ?
}

\author{
Thierry Ménissier
}

P.U.F. | Revue de métaphysique et de morale

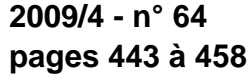

ISSN 0035-1571

Article disponible en ligne à l'adresse:

http://www.cairn.info/revue-de-metaphysique-et-de-morale-2009-4-page-443.htm

Pour citer cet article :

Ménissier Thierry, « La légitime défense, hier et aujourd'hui : le « résidu réaliste » du droit international ? », Revue de métaphysique et de morale, 2009/4 n64, p. 443-458. DOI : 10.3917/rmm.094.0951

Distribution électronique Cairn.info pour P.U.F.. (c) P.U.F.. Tous droits réservés pour tous pays.

La reproduction ou représentation de cet article, notamment par photocopie, n'est autorisée que dans les limites des conditions générales d'utilisation du site ou, le cas échéant, des conditions générales de la licence souscrite par votre établissement. Toute autre reproduction ou représentation, en tout ou partie, sous quelque forme et de quelque manière que ce soit, est interdite sauf accord préalable et écrit de l'éditeur, en dehors des cas prévus par la législation en vigueur en France. II est précisé que son stockage dans une base de données est également interdit. 


\title{
La légitime défense, hier et aujourd'hui : le « résidu réaliste » du droit international?
}

\begin{abstract}
RÉSUMÉ. - À partir de l'article 51 de la Charte des Nations unies, ce texte examine quelques problèmes posés dans l'histoire des idées politiques par l'argument de la légitime défense dans le cadre de la justification politique de la guerre et en fonction de la réflexion philosophique sur le thème de la guerre juste. Il envisage analytiquement cet argument, en mettant en lumière l'importance qu'il accorde à la souveraineté nationale, puis l'ambiguïté de la notion de «droit naturel» des États sur laquelle il se fonde. Or, conçue de manière "réaliste", au lieu d'être vectrice de désordres, la légitime défense offre paradoxalement le moyen d'une intégration des relations internationales, dans le système westphalien comme dans le système onusien. L'article aborde enfin la pertinence philosophique et politique de la «légitime défense préventive».
\end{abstract}

ABSTRACT. - Starting from the analysis of the article 51 of the United Nations Charter, this text examines many problems posed by the self-defense argument in the context of the political justification of war and in accordance with the philosophical reflexion about the just war. This argument is analytically considered, and the relevance of national sovereignty is underlined at first, and at second the ambiguity of "natural right », on which this argument is based. When the self-defense is realistically conceived, it makes any disturbance, but provides the way of international relations integration, in the Westphalian system or in the United Nations system. Lastly, the article tackles the philosophical and political value of self-defense as preemptive war.

«[...] On distingue précisément les gouvernements tyranniques des autres selon la manière dont ils utilisent la situation exceptionnelle pour la rendre permanente au lieu de viser le retour à la normalité et au respect du droit.»

Pierre Hassner

L'argument de la légitime défense a longtemps constitué dans le droit international public moderne et contemporain la forme paradigmatique d'un usage autorisé de la force. Aussi occupe-t-il une place de choix dans le catalogue des raisons invoquées pour justifier la guerre ou pour construire une réflexion philo- 
sophique à propos de la «guerre juste». Sous sa forme contemporaine, c'està-dire tel que l'entend l'article 51 de la Charte des Nations unies du 26 juin 1945, il constitue moins une exception au principe général de non-recours à la force dans les relations entre États qu'un cas d'espèce de la régulation juridique, et ses conditions d'expression sont assez précisément codifiées. La Charte distingue de plus la légitime défense individuelle et collective: la première concerne l'agression dont est victime un État souverain de la part d'un autre État souverain; la seconde évoque la faculté pour un État non directement agressé d'intervenir au nom d'accords de défense le liant au pays agressé.

Une telle forme d'intervention fut revendiquée par les États-Unis successivement au Liban, au Viêt Nam, à Saint-Domingue et au Nicaragua, et également par l'URSS pour justifier ses interventions à Prague en 1969 et en Afghanistan en 1979. D'autres exemples historiques peuvent être évoqués, dans lesquels des États ont eu recours à l'article 51, sans que l'instance onusienne valide systématiquement ce recours : dans la plupart des cas, il s'agit assez nettement d'agression délibérée et planifié de la part d'États développant une politique d'expansion ou de régulation de territoires qu'ils tenaient sous leur contrôle ${ }^{1}$. À tel point que, concernant l'article 51, on est quasiment tenté, sans forcer le paradoxe, de le ranger parmi les armes offensives dont disposent les États pour parvenir à leurs fins.

À la lumière d'exemples historiques qui ne sont même plus ambigus, mais éloquents quant à l'instrumentalisation permanente de l'argument par les États puissants, mon intention est d'examiner les relations complexes qui se nouent nécessairement entre la légitime défense et l'idée de souveraineté nationale ou étatique - en fonction de l'hypothèse selon laquelle la première représente le «résidu réaliste» que le droit international n'a, pour l'heure, jamais réussi à dissoudre. Mon analyse prendra d'abord pour objet la forme standard de la légitime défense, puis son évolution à la lumière des transformations de la souveraineté des États dans le contexte de la mise en œuvre d'une justice internationale, et en fonction des tendances plus ou moins récentes à la constitution d'une «légitime défense préventive » (autrefois évoquée par Israël, successivement en 1967, 1975 et 1981, à présent utilisée par les États-Unis vis-à-vis de son opinion publique interne pour justifier l'invasion de l'Irak), dont il est nécessaire de se

1. Voir par exemple: l'intervention de la France et du Royaume-Uni en Égypte en 1956, dite «affaire de Suez » 1'attaque aérienne anglaise au Yemen en mars 1964 ; le bombardement par Israël du réacteur nucléaire irakien Osirak le 7 juin 1981 ; l'opération «Paix en Galilée » d'Israël au Liban en 1982; l'intervention de l'Afrique du Sud contre l'Angola en 1985; le raid aérien des États-Unis sur Tripoli et Benghazi du 15 avril 1986; la destruction en vol d'un avion civil d' Iran Air par le navire US Vincennes au-dessus du détroit d'Ormuz le 3 juillet 1988; l'incident aérien entre des avions militaires américains et libyens en haute mer le 4 janvier 1989; les bombardements états-uniens au Soudan et en Afghanistan en 1998 après les attentats contre des ambassades en Afrique. 
demander si elle constitue une aberration dans le cadre du système onusien, ou bien s'il s'agit de l'évolution logique d'un argument équivoque dès son origine.

\section{LA LÉGITIME DÉFENSE, ARGUMENT JURIDIQUE DANS LE SYSTÈME DE L'ONU}

Il est dans un premier temps tentant d'affirmer qu'avec la mise en œuvre de la Charte de San Francisco, le thème de la légitime défense est devenu un argument juridique à part entière, cela dans un contexte au sein duquel le droit international public a acquis une nouvelle et plus complète normativité. Auparavant, il recouvrait sans les dissiper nullement les équivoques de la notion politique de légitimité. Celle-ci désigne ce qui apparaît juste ou fondé à un sujet agissant, et partant ce qui l'autorise à agir, ou ce dont il s'autorise pour agir. Cette notion engage donc un principe de justification de l'action qui s'entend de manière logique et axiologique. Avant l'instauration du système onusien, ce principe se confondait avec la raison d'être des États, dans une dimension où ils sont seuls juges du point où, comme le laissait entendre Hegel, il est raisonnable de placer l'offense ${ }^{2}$. Notre hypothèse est que, même sous sa forme contemporaine de l'article 51, la légitime défense n'échappe pas à l'équivocité de la notion de légitimité; et qu'une telle manière de l'appréhender jette un aperçu sur le droit international public qui le qualifie en profondeur, ou indique le registre qui est le sien en regard de sa «matière première », à savoir les relations internationales.

Ce sont les éléments constitutifs de l'article 51 qui nous invitent à une telle représentation des choses. Il affirme en effet:

Aucune disposition de la présente Charte ne porte atteinte au droit naturel de légitime défense, individuelle ou collective, dans le cas où un Membre des Nations unies est l'objet d'une agression armée, jusqu'à ce que le Conseil de sécurité ait pris les mesures nécessaires pour maintenir la paix et la sécurité internationales. Les mesures prises par les Membres dans l'exercice de ce droit de légitime défense sont immédiatement portées à la connaissance du Conseil de sécurité et n'affectent en rien le pouvoir et le devoir qu'a le Conseil, en vertu de la présente Charte, d'agir à tout moment de la manière qu'il juge nécessaire pour maintenir ou rétablir la paix et la sécurité internationales.

2. Hegel, Principes de la philosophie du droit, § $334:$ : «...] La question de savoirs [quels dommages] seraient à envisager comme une rupture déterminée des traités ou une atteinte à la reconnaissance et à l'honneur reste une chose indéterminable en soi, du moment qu'un État peut placer en chacune de ses singularités son infinité et son honneur, etc. », trad. J.-L. Vieillard-Baron, Paris, Flammarion, «GF », 1999, p. 385. 
La Charte entend exactement par «légitime défense» l'emploi de la force autorisée par la situation de péril dans laquelle se trouve un État quant à sa survie en tant qu'entité politique, quant à l'intégrité de son territoire et quant à la sécurité de ses citoyens. Affirmée dans le contexte de la Charte, une telle autorisation ne relève pas du tout d'une suspension du droit, mais au contraire de la thématisation juridique d'un usage légitime, raisonnable et temporaire de la force: ainsi son exercice doit-il être proportionné à l'agression subie, et la riposte, relativement immédiate, ces deux conditions évoquant d'ailleurs les clauses équivalentes dans le droit civil. De surcroît, le recours à la force n'est estimé légitime que le temps nécessaire à l'Organisation, représentée par le Conseil de sécurité, pour intervenir afin de rétablir l'ordre. Un point supplémentaire mérite d'être relevé, du fait qu'il évoque le principe censé justifier l'usage de la force: le texte stipule que le recours à la légitime défense est fondé sur un «droit naturel». La version en langue anglaise porte «inherent right», selon une expression qu'il paraît judicieux de traduire en français par l'expression «droit constitutif», et qui, comme telle, renvoie à la volonté étatique, et plus exactement au principe qui constitue le cœur de celle-ci, à savoir la souveraineté. Traditionnellement entendue comme "puissance absolue et perpétuelle d'une république $»^{3}$, celle-ci se définit à la fois comme le principe des pouvoirs de l'État et comme la règle de leur hiérarchie, ces deux dimensions permettant leur intégration fonctionnelle au sein de la machine étatique. Envisagée comme un faisceau de prérogatives, la souveraineté désigne plusieurs capacités différentes, telles que celle de faire librement les lois, de déclarer la guerre, d'instituer des magistrats et des officiers (et de veiller à l'exercice de leur tâche), de gracier en dernier recours, pour reprendre schématiquement les «marques de souveraineté » autrefois énumérées par Bodin ${ }^{4}$. Dans l'ordre international, la souveraineté est entendue comme la capacité d'un État à suivre sa propre ligne de conduite, elle désigne l'autonomie ou, caractérisée négativement, le fait de ne pas être sous la tutelle d'un autre État. Une telle approche qualifie pour ainsi dire le régime du droit international: puisque les relations internationales sont animées par le jeu de la volonté diverse des États, ceux-ci en sont les sujets, et ils sont les auteurs de la codification juridique afférente. De cette multiplicité des sujets/auteurs du droit international découlent certaines conséquences. Ainsi, celle de la normativité spéciale de ce droit, dans lequel les sanctions sont nécessairement d'une nature très différente de celles que connait le droit civil; dans ce cas, la discipline imposée aux sujets par le

3. Jean Bodin, Les Six Livres de la République, livre I, chap. 8, Paris, Fayard, 1986, tome I, p. 179.

4. Ibid., I, 10, pp. 306-341. 
droit est très différente, car ceux-ci ne sont pas directement les auteurs de la norme ${ }^{5}$.

\section{PARADOXES DU DROIT NATUREL}

Cependant, au lieu de simplifier les choses, l'examen plus attentif de la notion de «droit naturel» mentionnée comme fondement du légitime recours à la force défensive les rend problématiques. Il est même permis de se demander si le droit naturel des États à se défendre en cas d'agression constitue un moyen favorable à la régulation juridique de l'ordre international, ou s'il représente un obstacle pour celle-ci. En effet, on peut mettre en lumière le fait que, dans l'histoire des relations interétatiques et dans celle de leur codification, il a constitué aussi bien un facteur de perturbation qu'un vecteur d'ordonnancement.

Un bref détour par l'histoire théorique de la notion de droit naturel nous renseigne. Dès l'origine, il existe en réalité deux versions du droit naturel, que l'on peut respectivement qualifier d'idéaliste et de réaliste, et qui se trouvent en radicale opposition. La première, dont l'origine remonte à la lointaine thématisation des «lois non écrites» par la pensée grecque, apparaît nettement dans la synthèse constituée par la doctrine philosophique de Cicéron, lorsque ce dernier, dans le contexte dramatique de la fin de la République romaine, en appelait à une structure d'ordre invisible qui permet aux individus livrés à la tyrannie de l'identifier et de la qualifier comme illégitime et injuste ${ }^{6}$. La version réaliste du droit naturel, quant à elle, s'exprime moins dans les traités philosophiques - sauf pour y être sévèrement critiquée, ainsi qu'on le voit dans le Gorgias ou dans $L a$ République de Platon - que dans les œuvres des écrivains politiques. Thucydide, en faisant parler les généraux du corps expéditionnaire envoyé par Athènes contre l'île de Mélos, en a donné une formulation célèbre, dans laquelle l'affirmation d'un «droit à la puissance» fondé en nature a comme conséquence la déduction de la nécessité pour le faible d'obéir aux forts, dans le cadre d'une réversibilité toujours possible des positions occupées par les uns et les autres. Tandis que les sénateurs méliens arguent de leur droit à demeurer neutres dans le conflit, ou à s'engager du côté qui leur paraît bon, et alors qu'ils ont invoqué pour fonder ce droit la version idéaliste du droit naturel, les Athéniens leur rétorquent :

5. Ce point est mis en valeur par Agnès LeJBowiCz dans Philosophie du droit international. L'impossible capture de l'humanité, Paris, PUF, 1999.

6. Cf. CicÉron, Des lois, I, 15, trad. Ch. Appuhn, Classiques Garnier, 1965, p. 225. 
Nous estimons, en effet, que du côté divin comme aussi du côté humain [...], une loi de nature fait que toujours, si l'on est le plus fort, on commande; ce n'est pas nous qui avons posé ce principe ou qui avons été les premiers à appliquer ce qu'il énonçait: il existait avant nous et existera pour toujours après nous, et c'est seulement notre tour de l'appliquer, en sachant qu'aussi bien vous ou d'autres, placés à la tête de la même puissance que nous, vous feriez de même. Si bien que, pour ce qui est de la divinité, il est fort naturel que nous n'ayons pas peur d'être en désavantage ${ }^{7}$.

Dans la vision thucydidéenne de l'histoire, le «droit à la puissance» commande l'expansion territoriale des États et par suite conditionne la forme des relations internationales. L'exilé de Skapté Hylè paraît avoir considéré qu'il est déterminé par des motifs pathologiques : l'impérialisme des États (leur tendance à l'archê) est la conséquence de l'impossibilité des gouvernants à maîtriser leur propre orientation politique. Les mots mis dans la bouche d'Alcibiade, le brillant traître, sont éloquents à cet égard ${ }^{8}$. En instaurant par-delà les époques un dialogue entre les grandes œuvres, certains auteurs, comme Tite-Live, ont commenté les modalités de ce «droit à la puissance». Campant la scène dans le Sénat carthaginois vaincu à l'issue de la deuxième guerre punique, l'historien latin permet au personnage d'Hannibal de révéler de quelle nécessité interne relève son expression :

Aucun État puissant ne peut s'accommoder de la paix; s'il ne fait pas la guerre à l'étranger, il trouve chez lui la guerre civile, comme ces êtres qui paraissent insensibles aux maladies extérieures mais succombent au trop-plein de forces qui est en eux ${ }^{9}$.

Il existerait donc une fragilité paradoxale de la puissance inactive, dont la violence des humeurs dégraderait le trop-plein de santé.

Dans la modernité, la version idéaliste paraît avoir cédé le pas face à la réaliste, ou du moins leurs lignes se sont brouillées. Leo Strauss a fourni de l'histoire intellectuelle du droit naturel moderne une interprétation qui converge dans un tel sens ${ }^{10}$. Il s'agit en effet pour le philosophe américain d'origine allemande d'analyser les conditions théoriques et les enjeux moraux, politiques et philoso-

7. Les généraux athéniens aux Méliens dans THucydide, Histoire de la guerre du Péloponnèse, Livre V, 105, 2, trad. J. de Romilly, Paris, Robert Laffont, 1990.

8. «Il nous est impossible de régler, comme on fait d'un domaine, l'extension de notre empire, mais au point où nous nous sommes mis, force nous est, ici, d'ourdir des menaces, là, de ne pas céder, car le risque est pour nous de tomber, le cas échéant, sous l'empire d'autrui, si nous n'en exercions pas nous-mêmes un sur d'autres ", Histoire de la guerre du Péloponnèse, VI, 18, 3, traduction citée.

9. Hannibal dans TrTe-Live, Histoire de Rome depuis sa fondation (Ab Urbe condita), XXX, 44, trad. A. Flobert, Flammarion, «GF», tome IV, 2001, p. 463.

10. Leo StRauss, Natural Right and History [1953], trad. Droit naturel et histoire par M. Nathan et E. de Dampierre, Paris, Plon, 1954 ; Flammarion, «Champs », 1986. 
phiques du droit naturel moderne en regard de sa rupture avec la version idéaliste classique. Dans sa critique de la Modernité, toutefois, Strauss ne met pas en question l'existence effective d'un droit naturel moderne : il estime que la modernité a confondu les caractères distinctifs des deux versions. Ainsi soumet-il à rude épreuve les doctrines de Hobbes, de Locke et de Rousseau. Pour Strauss, l'inflexion individualisante du droit naturel moderne a littéralement ruiné la possibilité de disposer d'un critère d'évaluation du juste et de l'injuste, car ce critère ne saurait se déterminer que dans un ordre transcendant l'individualité, voire seulement dans la représentation de la nature comme «kosmos», c'està-dire comme monde harmonieusement hiérarchisé dans lequel chaque être occupe la place qu'il doit occuper. Or, au sein des doctrines modernes appelées à jouer le rôle de standards philosophiques de l'ordre juridique, estime-t-il, il ne reste pour déterminer le droit naturel que la puissance (potentia, power) portée par les individus singuliers. Les œuvres de Hobbes ${ }^{11}$ et de Spinoza ${ }^{12}$ attestent incontestablement d'une telle inflexion; dans une certaine mesure, il paraît également possible de la déceler dans celle de Locke ${ }^{13}$.

11. HobBes, Léviathan, chap. XIV, trad. F. Tricaud, Paris, Sirey, 1971, p. $128:$ «Le droit de nature [right of nature], que les auteurs appellent généralement jus naturale, est la liberté qu'a chacun d'user comme il le veut de son pouvoir propre, pour la préservation de sa propre nature [is the Liberty each man hath, tu use his own power, as he will himselfe, for the preservation of his own Nature], autrement dit de sa propre vie, et en conséquence de faire tout ce qu'il considérera, selon son jugement et sa raison propres, comme le moyen le mieux adapté à sa fin. »

12. SpInOZA, Traité théologico-politique, chap. XIV, trad. Ch. Appuhn, tome II des Euvres de Spinoza (reéd. Flammarion, «GF», 1965), pp. 261-262 : «Par droit et institution de la nature, je n'entends autre chose que les règles de la nature de chaque individu, règles suivant lesquelles nous concevons chaque être comme déterminé à exister et à se comporter d'une certaine manière. Par exemple, les poissons sont déterminés par la nature à nager, les grands poissons à manger les petits, en vertu d'un droit naturel souverain. Il est certain en effet que la nature considérée absolument a un droit souverain sur tout ce qui est en son pouvoir, c'est-à-dire que le droit de la nature s'étend aussi loin que s'étend sa puissance [jus naturae eo usque se extendere, quo usque ejus potentia se extendit] ; car la puissance de la nature est la puissance même de Dieu qui a sur toutes choses un droit souverain [naturae enim potentia ipsa Dei potentia est, qui summum jus ad omnia habet]. »

13. LocKE, Second traité du gouvernement civil, § 181, trad. J.-F. Spitz, Paris, PUF, 1994, pp. 133-134: «Certes, dans toute guerre, l'usage de la force s'accompagne ordinairement de dommages, et l'agresseur manque rarement de nuire aux biens lorsqu'il recourt à la force contre les personnes de ceux à qui il fait la guerre; et pourtant c'est l'usage de la force seul qui place un homme dans l'état de guerre. Soit que l'on recoure à la force pour commettre une injustice, soit que, l'ayant commise sans violence et par ruse, on refuse ensuite d'en faire réparation, et qu'on use de la force pour la soutenir (ce qui revient au même que de l'avoir commise par force), c'est le recours injuste à la force qui institue la guerre. Car, que l'on s'introduise chez moi par effraction et qu'on m'en expulse par la violence, ou que, après s'y être introduit paisiblement, on m'empêche d'y entrer, cela revient au même si l'on suppose que nous sommes dans un état où nous n'avons pas de juge commun sur terre auquel je puisse faire appel et auquel nous soyons les uns et les autres obligés de nous soumettre; or, telle est bien la situation dont je parle ici. C'est donc l'usage injuste de la force qui met un homme en état de guerre avec un autre; et par là, celui qui est coupable perd tout droit sur sa vie. En abandonnant la raison, qui est la règle donnée pour régir les rapports d'homme à homme, et en recourant à la force qui est la voie des bêtes, on s'expose à être détruit par celui contre qui on emploie 
L'interprétation straussienne de l'histoire du droit naturel est fort suggestive pour appréhender l'argument de la légitime défense dans le droit international. En effet, si la reconnaissance de cet argument semble constituer un point paradoxal dans le dispositif de la Charte de San Francisco, du fait qu'il reconnaît la possibilité de recourir à la violence en tant qu'expression d'une forme de légitimité dont la source, émanant des États particuliers, demeure à jamais étrangère au système du droit international, en réalité, parce qu'il fait référence à un droit de nature considéré comme une puissance ou un pouvoir constitutifs de l'État, cette reconnaissance apparaît comme logique et nécessaire. Son usage n'en est pas pour autant simplifié : ainsi que je l'ai souligné plus haut, les recours contemporains à l'argument de la légitime défense de la part d'États agressifs ou expansionnistes attestent de son caractère intrinsèquement problématique.

Mais en ce point un certain renversement est susceptible de se produire. Au lieu d'être uniquement vectrice de désordres, la légitime défense permet en effet une intégration des relations internationales, si du moins on les considère à l'aune des logiques de souveraineté déployées par les États. Elle se trouve de fait aisément intégrable dans le cadre d'une théorie de l'équilibre des pouvoirs. C'est la leçon qu'on peut tirer de deux modèles historiques différents.

D'une part, les doctrines internationalistes inspirées par l'esprit des traités de Westphalie, qui réglèrent l'équilibre des souverainetés nationales $d u \mathrm{XVII}^{\mathrm{e}}$ au $\mathrm{XIX}^{\mathrm{e}}$ siècle, offrent le moyen de comprendre ce paradoxe. Dans son «Examen de conscience sur les devoirs de la royauté», Fénelon estimait par exemple que si certaines conditions sont remplies, les nations voisines d'un État puissant ont le droit de lui résister en entreprenant une campagne agressive:

Quand une puissance monte à un point que toutes les autres puissances voisines ensemble ne peuvent plus lui résister, toutes ces autres sont en droit de se liguer pour prévenir cet accroissement, après lequel il ne serait plus temps de défendre la liberté commune. Mais, pour faire légitimement ces sortes de ligues qui tendent à prévenir un trop grand accroissement d'un État, il faut que le cas soit véritable et pressant: il faut se contenter d'une ligue défensive, ou du moins ne la faire offensive qu'autant que la juste et nécessaire défense se trouvera renfermée dans les desseins d'une agression; ou encore même faut-il toujours dans les traités de ligues offensives, poser des bornes précises, pour ne détruire jamais une puissance sous prétexte de la modérer.

Cette attention à maintenir une espèce d'égalité et d'équilibre entre les nations voisines est ce qui en assure le repos commun. À cet égard, toutes les nations voisines et liées par le commerce font un grand corps et une espèce de communauté. [...]

Les ligues défensives sont justes et nécessaires, quand il s'agit véritablement de prévenir

la force, au même titre que n'importe quelle bête sauvage et malfaisante qui menacerait son existence.» 
une trop grande puissance qui serait en état de tout envahir. Cette puissance supérieure n'est donc pas en droit de rompre la paix avec les autres États inférieurs, précisément à cause de leur ligue défensive, car ils sont en droit et en obligation de la faire ${ }^{14}$.

Vattel admettait de son côté la nécessité de reconnaître un «droit de sûreté » corrélatif des situations internationales dans lesquelles un État donne des signes d'impérialisme :

§ 42. Dès qu'un État a donné des marques d'injustice, d'avidité, d'orgueil, d'ambition, d'un désir impérieux de faire la loi, c'est un voisin suspect dont on doit se garder; on peut le prendre au moment où il est sur le point de recevoir un accroissement formidable de puissance, lui demander des sûretés, et s'il hésite à les donner, prévenir ses desseins par la force des armées. [...]

$\S 49$. Du droit de sûreté. C'est en vain que la nature prescrit aux nations, comme aux particuliers, le soin de se conserver, celui d'avancer leur propre perfection et celle de leur État, si elle ne leur donne pas le droit de se garantir de tout ce qui peut rendre ce même soin utile. Le droit n'est autre chose qu'une faculté morale d'agir, c'est-à-dire de faire ce qui est moralement possible, ce qui est bien et conforme à nos devoirs. Nous avons en général le droit de faire tout ce qui est nécessaire à l'accomplissement de nos devoirs. Toute nation, comme tout homme, a donc le droit de ne point souffrir qu'un autre donne atteinte à sa conservation, à sa perfection et à celle de son État, c'est-à-dire de se garantir de toute lésion: et ce droit est parfait, puisqu'il est donné pour satisfaire à une obligation naturelle et indispensable. Lorsqu'on ne peut user de contrainte pour faire respecter ce droit, l'effet en est très incertain. C'est ce droit de se garantir de toute lésion, que l'on appelle droit de sûreté.

$\S 50$. Il produit le droit de résister. Le plus sûr est de prévenir le mal, quand on le peut. Une nation est en droit de résister au mal qu'on veut lui faire, d'opposer la force, et tout moyen honnête, à celle qui agit actuellement contre elle, et même d'aller au-devant des machinations, en observant toutefois de ne point attaquer sur des soupçons vagues et incertains, pour ne pas s'exposer à devenir elle-même un agresseur injuste.

$\S 51$. Et celui de poursuivre la réparation. Quand le mal est fait, le même droit de sûreté autorise l'offensé à poursuivre une réparation complète et à y employer la force s'il est nécessaire.

$\S 52$. Et le droit de punir. Enfin, l'offensé est en droit de pourvoir à sa sûreté pour l'avenir, de punir l'offenseur, en lui infligeant une peine capable de le détourner dans la fuite de pareils attentats, et d'intimider ceux qui seraient tentés de l'imiter. Il peut même, suivant le besoin, mettre l'agresseur hors d'état de nuire. Il use de son droit dans toutes ces mesures, qu'il en résulte du mal pour celui qui l'a mis dans la nécessité d'agir ainsi ; celui-ci ne peut en accuser que sa propre injustice ${ }^{15}$.

14. FÉNELON, «Examen de conscience sur les devoirs de la royauté » [1712], in Écrits et lettres politiques, Paris-Genève, Slatkine, 1981, pp. 81-82.

15. Emeric DE VATTEL, Le Droit des gens ou Principes de la loi naturelle appliqués à la conduite et aux affaires des nations et des souverains [1757], livre II, tome II, chap. 4. 
Ce droit de sûreté, engendré par la prescription naturelle de survivre qui est faite à toute collectivité nationale, légitime la résistance active, le recours à la force préventive, ainsi que la possibilité d'obtenir par ses propres moyens la réparation et la punition qu'on estime proportionnées au dommage et à l'offense subis. Ainsi entendu dans le monde westphalien, le droit de légitime défense engendre un équilibre des puissances favorable à un ordre international qui peut être vecteur de paix ${ }^{16}$.

D'autre part, la légitime défense paraît également receler un potentiel de régulation au sein même du système onusien. Avec la Charte de 1945, les nations voulurent se doter d'un organe de réglementation internationale efficace, capable d'apaiser les différends entre États sans pour autant, certes, annuler leurs prérogatives souveraines. C'est la raison pour laquelle dans le texte de la Charte la reconnaissance de la légitime défense - indéniable relief de la puissance nationale - s'est trouvée strictement encadrée au sein d'un chapitre vil qui dote le Conseil de sécurité d'un réel pouvoir d'action. Le nouveau système inaugurait de ce fait à propos du recours à la légitime défense un état d'esprit très différent de celui typique du monde westphalien: parce qu'elle est insérée dans une telle Charte, la légitime défense nationale est en réalité à concevoir comme l'expression de la volonté commune des États. Dans cette perspective s'explique notamment la restriction temporelle dans l'usage légitime de la force, considérée pertinente uniquement le temps nécessaire pour le Conseil de sécurité de prendre les mesures afin de maintenir la paix et la sécurité internationales. Le recours à la puissance nationale se voit donc légitimé pour autant seulement qu'il se trouve intégré à la régulation internationale, décidée, elle, après une délibération collective. La Charte de San Francisco vise en quelque sorte à dompter la sauvagerie naturelle de la puissance étatique, à partir de l'affirmation par l'article $2 \mathrm{du}$ postulat fondamental et inédit de l'égalité de souveraineté de tous les États membres, quelle que soit leur puissance. Le modèle ainsi engendré gagne à être appréhendé en termes de « sécurité collective ${ }^{17}$.

\section{III. ÉTERNEL RETOUR DU RÉALISME?}

Quelle réalité une telle construction donne-t-elle à l'espoir de dépasser le paradoxe initial ? Quelle possibilité existe dans les faits pour échapper à la contradic-

16. Voir sur ces positions les réflexions de Peter HAGGENMACHER, «L'État souverain comme sujet du droit international, de Vittoria à Vattel », Droits, n 16,1993 , pp. 11-20.

17. Voir Serge SuR, article «Sécurité collective», in Th. de Montbrial et J. Klein, Dictionnaire de stratégie, Paris, PUF, 2000, pp. 305-309. 
tion effective d'une régulation juridique internationale empruntant la voie de la logique de souveraineté nationale? À certains égards, il convient de parler d'un impossible dépassement, voire d'un «éternel retour» du réalisme ou de la politique de puissance des États. Ce qui était vrai dans le monde bipolaire d'avant 1989 ne l'est pas moins dans la situation actuelle des relations internationales.

D'abord, dans le cadre onusien, la légitime défense a été articulée dans la situation de tension permanente entre les deux «supergrands ». C'est-à-dire que pendant quarante ans elle a réglé leur affrontement effectif, quoique la plupart du temps mené de manière indirecte. La légitime défense a dans ces conditions représenté le mode de régulation - ou du moins la matrice d'intelligibilité d'une situation conflictuelle qui ne disait pas son nom et qui concernait l'ordre international dans sa globalité. On pouvait le recevoir comme un argument aussi bien stratégique que juridique, mais qui devait être appréhendé sur le plan stratégique pour pouvoir être accepté comme juridique, car il rendait compréhensibles, sinon en partie tolérables, les actes de puissance émanant des deux Grands. Il est permis d'estimer que cette logique s'est trouvée parfaitement reflétée par l'emploi de l'argument de la légitime défense dans le cadre de la doctrine stratégique française de la dissuasion nucléaire. Celle-ci reposait en effet sur la manifestation par une puissance du «club nucléaire » de sa ferme volonté de répliquer à l'agression en déchaînant le feu. Raymond Aron ${ }^{18}$ et Pierre Hassner ${ }^{19}$ ont analysé les modalités de ce recours à la force, dont le ressort consiste à ériger la légitime défense en principe pour un système stratégique. Expression du droit naturel d'une nation à survivre, le déclenchement du feu nucléaire s'est voulu à la fois principiel et proportionné à l'attaque subie. On sait d'ailleurs que s'il fut conçu dans cet esprit général, l'arsenal militaire français a évolué depuis 1962, notamment en termes de variété des vecteurs, et cela en fonction d'un affinement de la doctrine dans le sens de la graduation de la riposte.

Ensuite, que devient la légitime défense dans le monde « unipolaire » d'aujourd'hui ? Fort logiquement, elle a évolué, mais toujours en tant qu'elle se trouve conditionnée par la puissance qui en sous-tend la possibilité. Cette évolution générale connaît depuis 2001 une inflexion fondamentale en fonction de la menace diffuse et constante du terrorisme international, puisque des organisations secrètes sont à présent capables de provoquer des violences considérables sans pourtant relever de la logique classique de la souveraineté des nations. Fruit de cette inflexion, l'argument de la légitime défense préventive (ou «préemptive»)

18. Cf. Raymond Aron, Paix et guerre entre les nations, Paris, Calmann-Lévy, 1962, chap. XIv, «De la stratégie de dissuasion», pp. 400-435.

19. Voir Pierre HASSNER, «On ne badine pas avec la force» [1971], in La Terreur et l'Empire. La Violence et la Paix, II, Paris, Le Seuil, 2003, pp. 235-266. 
a été invoqué par les États-Unis dans leurs campagnes contre l'Afghanistan en 2001 puis contre l'Irak en 2003. Le texte qui fut à cette occasion rédigé par la Maison Blanche mérite ici d'être cité dans sa version originale :

While the United States will constantly strive to enlist the support of the international community, we will not hesitate to act alone, if necessary, to exercise a right of selfdefence by acting preemptively against such terrorists, to prevent them from doing harm against our people and our country [...] The United States as long maintained the option of preemptive actions to counter a sufficient threat to our national security. The greater the threat, the greater is the risk of inaction - and the more compelling the case for taking anticipatoring action to defend ourselwes, even if uncertainly remains as to the time and place of the enemy's attack. To forestall or prevent such hostile acts by our adversaries, the United States will, if necessary, act preemptively ${ }^{20}$.

Dans l'esprit de cette justification de l'agression, la menace plus ou moins diffuse engendre le recours à l'affirmation d'un droit à l'autodéfense, qui lui-même conduit à la revendication de la légitimité d'une attaque préventive. Le Conseil de sécurité de l'ONU, par ses résolutions successives, a tour à tour accompagné $^{21}$ puis refusé ${ }^{22}$ les prises de positions états-uniennes.

Une telle évolution du modèle de la légitime défense ne saurait surprendre. Elle était d'ailleurs déjà envisageable dans le modèle westphalien, ainsi qu'en atteste la position exposée par Fénelon dans le texte cité plus haut, qui défend le bien-fondé de la légitime défense préventive d'une alliance d'États ligués contre un autre, susceptible de menacer la stabilité de l'ordre international ${ }^{23}$. À l'époque contemporaine, l'État d'Israël l'avait déjà illustrée lors de la guerre des Six-Jours de $1967^{24}$. Quoique l'on puisse penser de la légitimité des campagnes menées par les États-Unis (de nombreuses voix dans le monde se sont notamment élevées pour critiquer l'attaque de l'Irak alors dirigé par Saddam Hussein), la revendication de la légitime défense préventive, lorsqu'elle est employée dans

20. National Security Strategy of the United States of America, document publié par la Maison Blanche en septembre 2002 (source: site de la Maison Blanche, www.whitehouse.gov).

21. La résolution 1368 du 12 septembre 2001 reconnaissait en effet l'action des États-Unis en Afghanistan.

22. La campagne irakienne des États-Unis n'a en effet pas été accompagnée de l'accord du Conseil de sécurité. Cependant, la résolution 1379/01 tendait à engager les nations de l'Organisation derrière les États-Unis. Voir sur ce point Serge SuR, «Quelle légalité pour le conflit armé en droit international ?», Cités, no 24/2005, pp. 103-117.

23. Voir également le rappel des thèses classiques sur la guerre préventive par Gregory M. REICHBERG dans son article «Jus ad Bellum», in Larry May (ed.), War. Essays in Political Philosophy, Cambridge University Press, 2008, pp. 25-27.

24. Cf. Michael WALzER, Guerres justes et injustes [seconde édition augmentée 1992], trad. Paris, Belin, 1999, pp. 130-135: Walzer envisage cet exemple comme le cas de figure d'une forme d'«anticipation » qu'il juge légitime. 
les conditions d'une menace effective, ne modifie nullement ni les règles communément admises ni les raisons classiquement invoquées lorsqu'on parle du jus ad bellum. Ces cas de figure se présentent comme ce que des auteurs, dans une expression heureuse, ont récemment nommé une «contre-violence unilatérale ${ }^{25}$. Quant à la difficulté d'évaluer la menace effective justifiant de manière indiscutable le recours à l'agression, elle constitue bien entendu un des points centraux du débat tout en n'étant rien moins que nouvelle, un auteur aussi avisé des choses de la guerre que Clausewitz l'avait déjà soulignée ${ }^{26}$.

Cette évolution conduit-elle cependant à conclure à la faillite voire à la caducité du système onusien ? Il semblerait qu'il connaisse en tout cas une crise grave, puisque rien ne semble empêcher le dernier supergrand d'agir tout seul ou à la tête des alliances politico-militaires qu'il parvient à contracter, et de mener les guerres qu'il entend mener au nom de ses intérêts nationaux. Et dans un contexte tel que celui de l'après-11 Septembre, l'article 51 de la Charte onusienne ne prend-il pas une tout autre dimension, en se transformant en obstacle infranchissable pour l'établissement d'une régulation juridique internationale? Une chose est en effet, ainsi que le fait Walzer, de reconnaître le bien-fondé de la légitime défense préventive dans le cas de l' «anticipation » israélienne en 1967, une autre est de revendiquer la même position dans le contexte de l'action d'une puissance désormais unique par sa suprématie militaire mondiale. Dans le premier cas, il s'agit de juger la réaction préventive d'un État dont la force demeure proportionnée à celle de ses rivaux, et dont l'étendue territoriale restreinte le place sous la menace effective d'une invasion rapide possible. Dans le second, il s'agit d'appréhender les conséquences d'un déploiement militaire sans équivalent, et loin des frontières nationales. On peut également considérer les choses sous l'angle des principes - réfléchir à ces problèmes revient en effet à affronter l'alternative qui gît depuis 1945 dans les dispositions onusiennes : l'article 51 exprime-t-il la béance constitutive du droit international, ou bien, telle une «ruse du droit», favorise-t-il l'expression d'une violence sous contrôle? Qu'il nous soit ici permis de nous inspirer de la belle analyse de Serge $\operatorname{Sur}^{27}$ : 1'examen attentif des engre-

25. Denys Simon et Linos-Alexander Sicilianos, «La contre-violence unilatérale. Pratiques étatiques et droit international », Annuaire français de droit international, volume 32, 1986, pp. 5378.

26. «Or, lorsque le combat n'a pas lieu, le seul fait de l'avoir rendu possible constitue déjà une réalité, un fait acquis qui aura immanquablement ses conséquences.» [...] «Un combat devient possible sur un point déterminé dès qu'on y forme des troupes, sans qu'il se produise pour autant réellement. Faut-il considérer cette simple possibilité comme une réalité, comme une chose effective? Certes oui, car elle le devient par ces conséquences, et des effets, quels qu'ils soient, ne peuvent jamais manquer de se produire», De la guerre, traduit de l'allemand par le lieutenant-colonel de Vatry, édition complétée et révisée par Jean-Pierre Baudet, Paris, G. Lebovici, 1989, p. 191.

27. Serge Sur, Le Conseil de sécurité dans l'après 11 Septembre, Paris, LGDJ, «Global Understanding Series », 2004. 
nages diplomatiques dans lesquels furent prises les interventions américaines en Afghanistan et en Irak permet d'établir que le Conseil de sécurité n'a jamais été complètement disqualifié par les évolutions récentes - à condition toutefois d'adopter une position « réaliste» sur la nature du droit international. À savoir, en reconnaissant que les relations internationales qui en constituent le matériau sont le reflet des jeux de la puissance interétatique, indomptables dans leur fond. Et par suite en admettant que le droit international, assorti du travail constant de la diplomatie, représente en quelque sorte l'«art prudentiel» dont il a toujours besoin, sa cheville ouvrière.

Ces développements nous inspirent deux séries de conclusions, l'une philosophique, l'autre politique.

Les conséquences d'ordre philosophique sont au nombre de quatre, et reflètent une appréhension du droit international inspirée par la considération «réaliste» de la présence indépassable dans le droit international de la légitime défense comme avatar de la puissance étatique. (1.) La légitime défense reflète la liberté des États ${ }^{28}$ - ce qui est vrai, bien entendu, même de sa forme d'agression préventive. (2.) Elle a fourni et fournit encore un mode de compréhension adéquat des opérations diplomatiques et militaires dans le cadre de la sécurité collective; à ce titre, et bien que cette remarque soit totalement paradoxale, elle constitue un facteur de limitation du recours à la guerre. (3.) Cependant, jusqu'à présent, les États ont toujours trouvé dans ce recours un principe de constitution dont on ne peut méconnaître la persistance - et l'argument de la légitime défense est le biais juridique qui en maintient la possibilité. (4.) L'ordre juridique mondial n'est rien d'autre que le degré de formalisation caractéristique des relations internationales à un moment donné en fonction de la compétition à laquelle se livrent les États. La dynamique propre de ces relations implique que la dialectique de la stabilité et de l'instabilité ne se trouve jamais définitivement réglée.

Les conséquences politiques sont elles aussi au nombre de quatre. (1.) La légitimité de la réaction militaire à une attaque (ou à une menace sévère) n'a jamais aboli la responsabilité des leaders politiques qui la décident, ni celle des officiers qui sur le terrain en déterminent l'exécution, et même tout au contraire : leur responsabilité est engagée du fait même qu'ils choisissent le recours au combat. Le caractère proportionné de la défense à l'attaque ou à la menace demeure un critère d'évaluation morale d'une grande valeur de publicité - un

28. Cf. Jean Combacau, «Pas une puissance, une liberté: la souveraineté internationale de l'État », Pouvoirs, n 67, 1993, pp. 47-58. Voir également Michael WALzer, «The Moral Standing of States», Philosophy and Public Affairs Reader on International Relations, Princeton University Press, 1985, pp. 165-194. 
référent externe qui est susceptible d'avoir des conséquences non seulement sur la forme des relations internationales, mais également, au sein des démocraties, sur le débat interne et sur la politique nationale. Aussi nous semble-t-il que le problème de l'évaluation d'une riposte graduée se posera toujours en tant que tel, et avec une acuité d'autant plus grande dans les guerres asymétriques contemporaines. Il n'est que de mentionner, à titre d'exemple (un exemple tout récent mais qui peut valoir comme cas d'espèce en la matière), les débats dans la communauté internationale autour de la «justesse» de la réponse d'Israël aux attaques du Hamas. Pour légitime qu'ait pu apparaitre la réaction militaire sous la forme de l'invasion de la bande de Gaza en décembre 2008, la destruction de la plupart des infrastructures locales, les tirs faisant des dizaines de victimes civiles et les agressions contre les institutions internationales installées sur place constituent des éléments de responsabilité accablants pour les chefs politiques et militaires israéliens, personne ne saurait sérieusement mettre cela en question. (2.) À la lumière de nos conclusions philosophiques, il faut reconnaître que même la forme préventive de la légitime défense peut se trouver justifiée logiquement et axiologiquement. D'autant plus si l'on admet, avec Pierre Hassner, qu'avec le 11 Septembre le monde est entré dans une nouvelle phase, où la peur et le désir de sécurité jouent un rôle $\operatorname{accru}^{29}$. La justification logique repose sur la nécessité naturelle qui est faite aux États de survivre; la justification axiologique peut se prévaloir de la volonté d'assurer la sécurité des populations, voire de défendre des valeurs telles que la liberté. Mais - surtout dans un contexte global - pour quelle efficacité ? Ne se trouve-t-elle pas par principe contre-performante dans l'optique d'un retour à la paix, et par suite en totale contradiction avec la volonté revendiquée d'instaurer un ordre légitime et sûr? (3.) Les récentes campagnes états-uniennes relèvent-elles de la guerre ou d'opérations de police internationale ? Elles ont du moins permis de poser à nouveaux frais les vieux dilemmes de la «guerre juste», sans fournir jamais l'espoir de les dépasser ${ }^{30}$. Et l'on peut réfléchir à la réponse qu'appelle cette question à la lumière de ce qui semble bien constituer un paradoxe de la puissance dans le monde unipolaire, tel qu'il est apparu à partir de 2001 : l'emploi de la force engendre ce qu'on a identifié comme la «responsabilité de protéger» qui est faite à la puissance mondiale dominante à l'égard des États plus faibles ou en danger. (4.) Dans le même temps, on peut subodorer à moyen terme, pour la légitime défense, le principe d'évolutions inouïes, et pour cela difficiles à anticiper: les nouvelles donnes politiques - par exemple l'affai-

29. Pierre HASSNER, «La signification du 11 septembre: divagations politico-philosophiques sur l'événement » [2002], in La Terreur et l'Empire, op. cit., pp. 383-402.

30. Cf. par exemple Chantal Delsol, La Grande Méprise. Justice internationale, gouvernement mondial, guerre juste, Paris, La Table ronde, 2004 ; et Monique CANTO-SPERBER, Le Bien, la guerre et la terreur. Pour une morale internationale, Paris, Plon, 2005. 
blissement de la forme même des États-nations au profit de nouvelles entités collectives, infranationales (des «nations » non reconnues dans leurs revendications de souveraineté; les régions dans leurs nouvelles acceptions) ou supranationales (par exemple l'Union européenne ou toute autre fédération à venir) - risquent dans les décennies qui viennent de conférer un tour totalement original au recours à cet argument.

Thierry MÉNISSIER Université Pierre Mendès France - Grenoble 2 